\title{
SEMI - GLOBAL MERGING OF DIGITAL SURFACE MODELS FROM MULTIPLE STEREOPAIRS
}

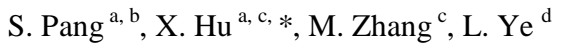 \\ ${ }^{a}$ Collaborative Innovation Center of Geospatial Technology, Wuhan University, Wuhan 430079, China - (psy, huxy)@whu.edu.cn \\ ${ }^{\mathrm{b}}$ School of Resource and Environmental Sciences, Wuhan University, Wuhan 430079, China \\ ${ }^{c}$ School of Remote Sensing and Information Engineering, Wuhan University, Wuhan 430079, China - mizhang@whu.edu.cn \\ ${ }^{\mathrm{d}}$ School of Educational Information Technology, Central China Normal University, Wuhan 430079, China - \\ ye_lizhi@mail.ccnu.edu.cn
}

Commission WG II/1, WG II/2

KEY WORDS: Digital surface model, Merging, Stereopairs, Semi - global optimization, Aerial images, Point cloud data

\begin{abstract}
:
The semi-global optimization algorithm, which approximates a global 2D smoothness constraint by combining several 1D constraints, has been widely used in the field of image dense matching for digital surface model (DSM) generation. However, due to occlusion, shadow and textureless area of the matching images, some inconsistency may exist in the overlapping areas of different DSMs. To address this problem, based on the DSMs generated by semi-global matching from multiple stereopairs, a novel semiglobal merging algorithm is proposed to generate a reliable and consistent DSM in this paper. Two datasets, each covering $1 \mathrm{~km}^{2}$, are used to validate the proposed method. Experimental results show that the optimal DSM after merging can effectively eliminate the inconsistency and reduce redundancy in the overlapping areas.
\end{abstract}

\section{INTRODUCTION}

Digital surface model (DSM) plays an important role in many applications, for example, object extraction and change detection. Thanks to the outstanding performance of semiglobal matching (SGM) (Hirschmüller, 2005; Hirschmüller, 2008), many researchers (Yastikli, et al., 2014; Ghuffar, 2016) tended to achieve the generation of DSMs with the SGM algorithm. However, considering that DSMs (also referred to as point cloud data) generated by SGM are single stereopair-based, as shown in Figure 1, certain inconsistencies may exist in the overlapping areas of different stereopairs. Furthermore, DSMs generated by SGM are of high density and redundancy, especially for the aerial images with multiple overlap. Thus, merging of these DSMs to obtain a consistent and accurate DSM is very necessary.

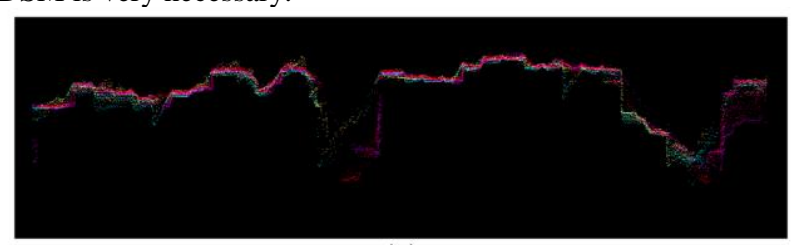

(a)

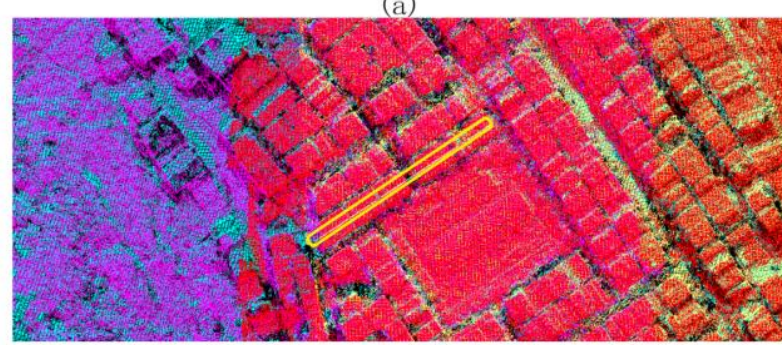

(b)
Figure 1. Inconsistencies in the point cloud data from different stereopairs, and different colors represent from different stereopairs; (a) Cross section of point cloud data in the yellow rectangle of (b); (b) Top view of point cloud data.

In the DSM merging, many scholars (Fratarcangeli, et al., 2016; Jaud, et al., 2016) tend to achieve it by using commercial software (e.g., INPHO, PhotoScan, MicMac and SURE). Meanwhile, some researchers cast the DSM merging as surface reconstruction problem, and screened poisson (Kazhdan and Hoppe, 2013), floating scale (Fuhrmann and Goesele, 2014), voronoi filtering (Amenta and Bern, 1999) and some probabilistic methods (Agrawal and Davis, 2001) were used to achieve a good surface reconstruction. In addition, Sadeq, et al. (2016) proposed a Bayesian approach to merge different DSMs from different sources. Furthermore, some researchers try to first divide the point cloud data into grids with regular intervals, then select an optimal point at each grid with the "winner takes all" strategy. For the images with good quality, the generated DSM is good. However, for the images with poor quality, considering that there is no consideration of spatial correlation, the generated DSM may still contain some outliers. And as Gong and Fritsch (2016) point out that merging of DSMs from multiple stereopairs can effectively remove outliers and further improve the quality of DSM generated by the SGM algorithm. Thus, based on the DSMs from multiple stereopairs generated by SGM, an automatic semi-global merging algorithm is proposed to obtain a more consistent and accurate DSM in this paper. This merging algorithm can not only remove the outliers in the point cloud data to obtain a reliable and consistent DSM, but also effectively reduce the redundancy in the overlapping areas of multiple DSMs.

\footnotetext{
* Corresponding author
} 
The rest of this paper is organized as follows. Section 2 first describes the basic idea of semi-global optimization. Section 3 presents the proposed merging algorithm with semi-global optimization for point cloud data from multi-stereopairs. Then, Section 4 displays the experimental results. Finally, conclusions are drawn in Section 5.

\section{SEMI - GLOBAL OPTIMIZATION}

Semi-global optimization is inspired by the SGM algorithm (Hirschmüller, 2008). Its basic idea is to approximate a global $2 \mathrm{D}$ smoothness constraint by combining several $1 \mathrm{D}$ constraints. It mainly consists of three parts: establishment of 3D cost matrix, multi-directional aggregation of costs, and acquisition of optimal surface, as shown in Figure 2. In the 3D cost matrix, the first two dimensions represent $X$ and $Y$ on the two - dimensional plane, and the third dimension represents the label. The values in each grid $(x, y, l)$ of the $3 \mathrm{D}$ cost matrix represent the cost of selecting the label $l$ on the 2D plane $(x, y)$. In general, the smaller the value, the greater the probability or possibility of selecting the label $l$. Then 8 or 16 directions of the dynamic programming algorithm are used for multi-directional aggregation of costs. During the dynamic programming process, it only needs to record the minimum cost of selecting each label in consideration of the cost of the label and the cost of the smooth constraint, but not need to record the optimal path. After the dynamic programming, the minimum cost of each grid will be accumulated to the accumulated cost matrix. And an optimal surface is calculated based on this accumulated cost matrix after all directions of the dynamic planning is completed. The optimal surface can be determined by the "winner takes all" strategy, that is, label of each grid on the 2D plane with the smallest value in the accumulated cost matrix is selected as the optimal label. Finally, a median filter is used to reduce the noise in the optimal surface.

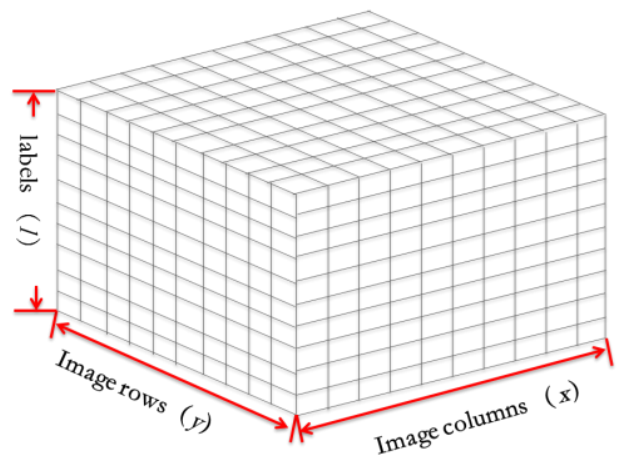

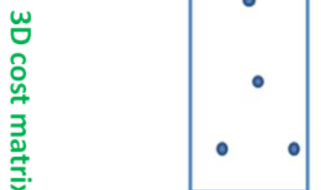

Original points

\section{SEMI - GLOBAL OPTIMIZATION FOR MERGING OF MULTIPLE DSMS}

Point cloud data obtained by SGM are single stereopair-based, and certain inconsistencies may exist in the overlap of different stereopairs. To obtain a consistent large range of DSM, a semiglobal optimization-based method is proposed to merge the point cloud data from multiple stereopairs in this paper, and details are as follows:

Step 1: Divided the point cloud data into different blocks

Considering that the amount of point cloud data may be too large to process at one time, the whole point cloud data is first divided into several DSMs with $1 \mathrm{~km}^{2}$ area each. The division could also facilitate the subsequent processing (e.g., object extraction and change detection).

\section{Step 2: Clustering of point cloud data}

The point cloud data in each kilometer is first assigned a grid index, and the grid size is set to a specific distance (e.g., $1 \mathrm{~m}$ ). Then, the points in each grid cell are clustered into several clusters by a certain height distance (e.g., $0.9 \mathrm{~m}$ ). The center and weight of each cluster are calculated. The center is represented by the mean of points in the cluster, and the weight is determined by the number of points in the cluster. The greater the number of points of the cluster, the greater the weight of each cluster. The process of clustering the points is shown in Figure 3.

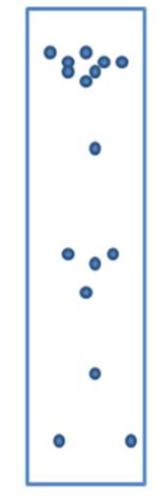

\section{Original points}

Figure 3. Process of clustering the points

The benefits of the clustering mainly have two aspects. On the one hand, it can reduce the number of candidate labels in the subsequent semi-global optimization and increase the speed. On the other hand, it can guide the optimization algorithm to select those labels composed of dense points by increasing the weight of the labels.

Step 3: Optimal DSM acquisition with semi-global optimization

Optimal DSM acquisition is achieved by semi-global optimization. The energy function which includes a data term and a smooth term, is expressed as

$$
\begin{aligned}
& l^{*}=\arg _{l} \min E(l)=\arg _{l} \min \left(E_{\text {data }}(l)+E_{\text {smooth }}(l)\right) \\
& E_{\text {data }}=-\sum \log V_{l}
\end{aligned}
$$

Figure 2. Flowchart of semi-global optimization 


$$
E_{\text {smooth }}=\sum\left\{\begin{array}{cc}
|d z| * 0.1 & |d z|<D Z_{1} \\
|d z| * 0.5 & D Z_{1} \leq|d z|<D Z_{2} \\
\left|D Z_{2}\right| & |d z| \geq D Z_{2}
\end{array}\right.
$$

where $\quad l=$ possible cluster centers for all grid cells,

$l^{*}=$ optimal cluster centers,

$V_{l}=$ weight of the cluster obtained by step 2 ,

$d z=$ height difference between two neighbor centers,

$D Z_{1}=$ threshold of height difference,

$D Z_{2}=$ threshold of height difference.

In general, natural surface and artificial object surface are mostly continuous and smooth. The aim of defining $D Z_{1}$ is to make the generated DSM surface smooth. Meanwhile, for the discontinuities, e.g., breaklines, defining $D Z_{2}$ is to set a larger constant penalty. In this paper, $D Z_{1}$ and $D Z_{2}$ are set to $1.0 \mathrm{~m}$ and $2.0 \mathrm{~m}$, respectively. After semi-global optimization, a median filter is used to reduce the noise in the generated DSM.

\section{Step 4: Progressive TIN-based DSM densifying}

After achieving the grid DSM obtained by the above steps, the DSM can be densified by progressive TIN algorithm. In the densification process, a sparse TIN is first derived from the grid DSM, then the points are progressively added to the TIN if they are below the defined thresholds. More details about the progressive TIN algorithm can be seen in (Axelsson, 2000).

\section{EXPERIMENTAL RESULTS}

In this paper, two datasets with $1 \mathrm{~km}^{2}$ area each are used for the experiments. Each dataset is composed of point cloud data from multiple stereopairs generated by the SGM algorithm (Hirschmüller, 2008). The stereopairs are from traditional aerial images obtained by DMC camera. The image size is 7,680 pixel $\times 13,824$ pixel, pixel size is $12 \mathrm{um}$, the focal length is $120 \mathrm{~mm}$, and forward lap and side lap are $65 \%$ and $35 \%$, respectively. corresponding orthophotos of the two datasets are shown in Figure 4.

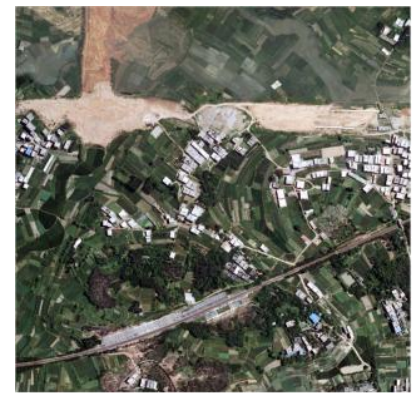

(a)

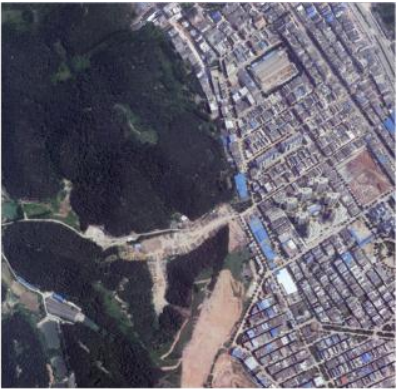

(b)
Figure 4. Corresponding orthophotos of the two datasets. (a) dataset 1 , (b) dataset 2 .

From Figure 4, it can be seen that dataset 1 is a typical suburban area with sparse housing and dense farmland. Dataset 2 is a complex area; the right half of the dataset is a typical main urban area with very dense houses, and the left half is a mountain covered by dense trees.

To visually represent the merging results of point cloud data from multiple stereopairs in this study, comparisons before and after merging were selected to be enlarged for dataset 1 and dataset 2. For dataset 1 , cross section and triangulation of the results are shown in Figures 5 and 6. For dataset 2, cross section and triangulation of the results are shown in Figures 7 and 8.

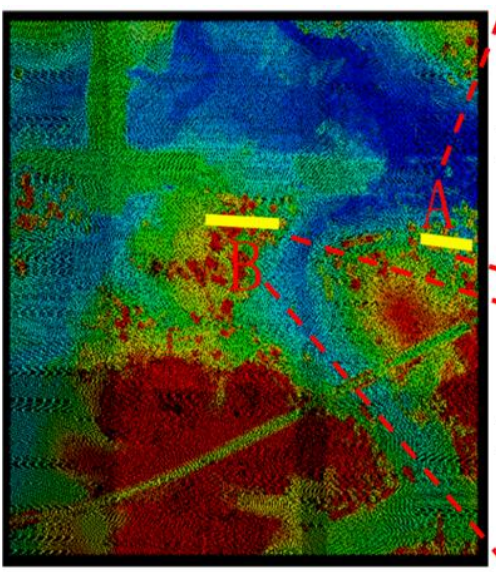

(a)

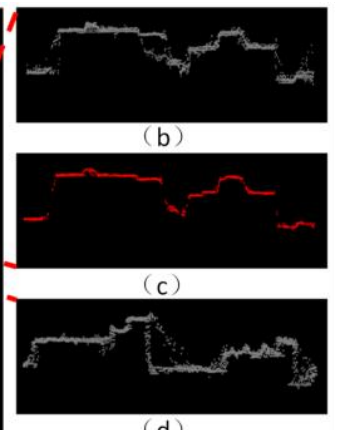

d)

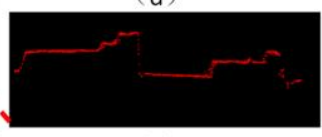

(e)
Figure 5. Cross section of dataset 1 before and after merging. (a) Overview of dataset 1, where Area A and Area B are two areas used to show the merging results; (b) Cross section of Area A before merging; (c) Cross section of Area A after merging; (d) Cross section of Area B before merging; (e) Cross section of Area B after merging.

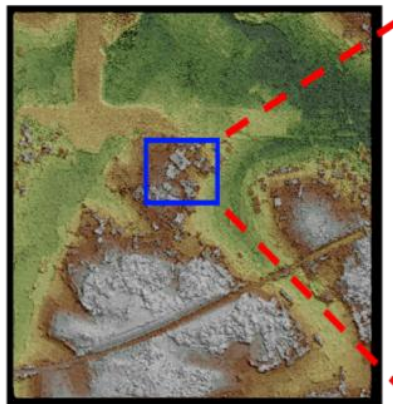

(a)

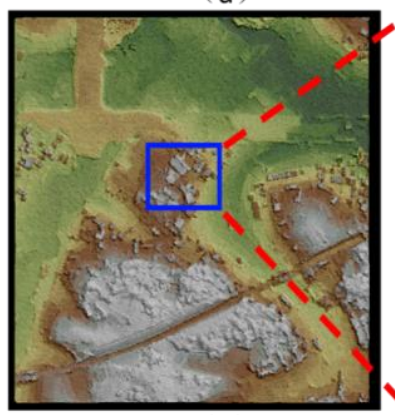

(c)

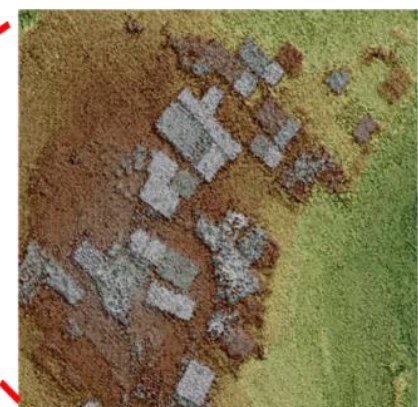

(b)

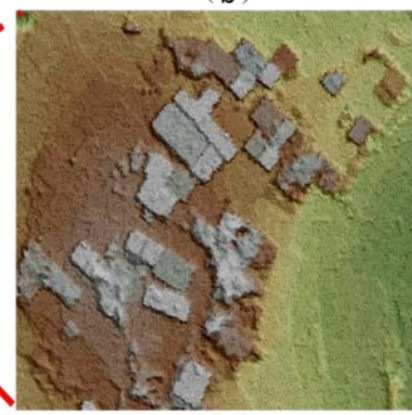

(d)
Figure 6. Triangulation of dataset 1 before and after merging. (a) Triangulation of dataset 1 before merging; (b) Enlarged subsets of dataset 1 before merging; (c) Triangulation of dataset 1 after merging; (d) Enlarged subsets of dataset 1 after merging. 


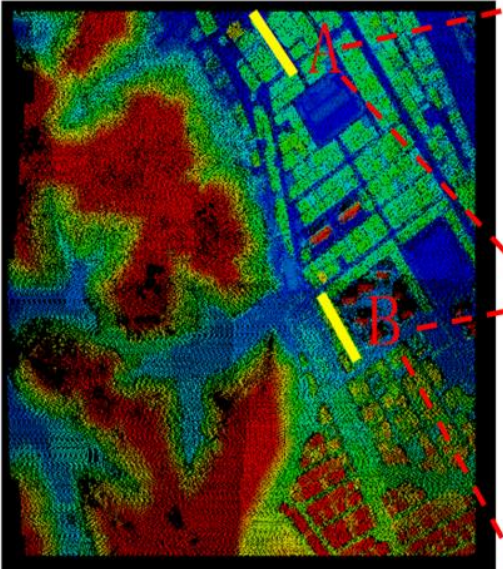

(a)

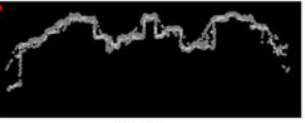

(b)

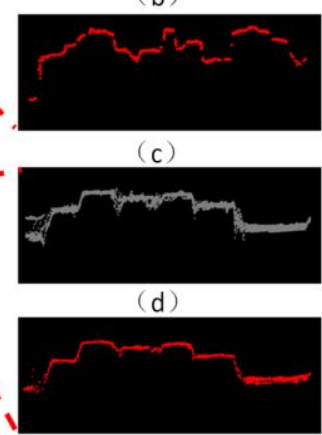

(e)

Figure 7. Cross section of dataset 2 before and after merging. (a) Overview of dataset 2, where Area A and Area B are two areas used to show the merging results; (b) Cross section of Area A before merging; (c) Cross section of Area A after merging; (d) Cross section of Area B before merging; (e) Cross section of Area B after merging.

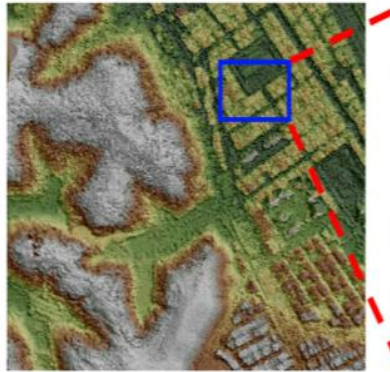

(a)

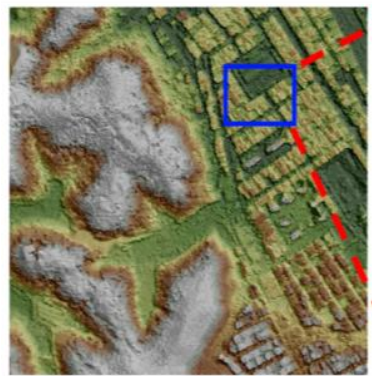

(c)

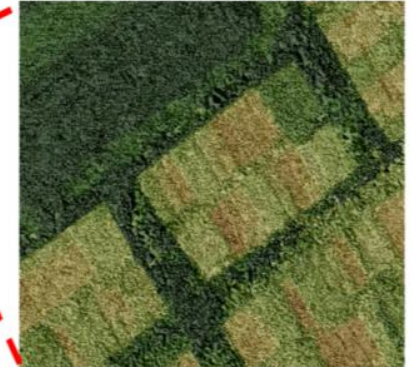

(b)

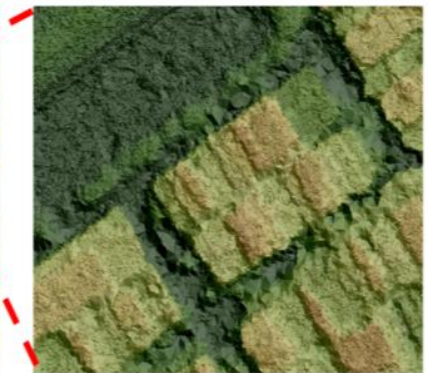

(d)
Figure 8. Triangulation of dataset 2 before and after merging. (a) Triangulation of dataset 2 before merging; (b) Enlarged subsets of dataset 2 before merging; (c) Triangulation of dataset 2 after merging; (d) Enlarged subsets of dataset 2 after merging.

Figure 5 and 7 show that multilayered phenomenon in those building boundaries is significantly reduced after merging, and the accuracy of point cloud data is improved. Furthermore, from Figure 6 and 8, it can be seen that many spikes are effectively removed after merging, and a smoothed surface is generated.

To better show the results of the proposed method, median of each grid cell is used to compare with our semi-global merging results. Here, the size of grid cell is set to the same as the proposed method. Furthermore, considering that points composed of median of each grid cell are sparse, TIN algorithm is also used to add the points which are below the defined thresholds.

Comparisons are shown in Figure 9, where Area 1 and Area 2 are from dataset1, and Area 3 is from dataset 2. The first row is the triangulation of the raw data, the second row is the results from median and TIN algorithm, and the third row is the results of the proposed method. From Figure 9, it can be seen that the triangulation of the raw data includes many burr-like noises, and the results of the median and TIN algorithm are improved obviously, but there is still a small amount of burr-like noise. The results from the proposed method are smooth and most of the burr-like noises have been further eliminated.

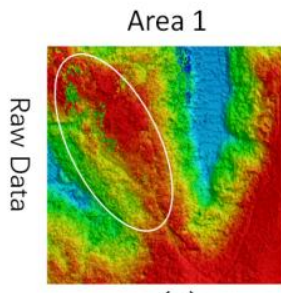

(a)

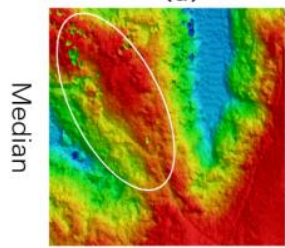

(d)

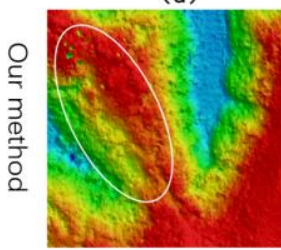

(g)

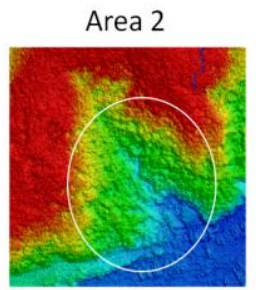

(b)

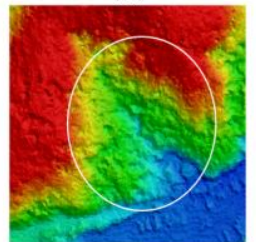

(e)

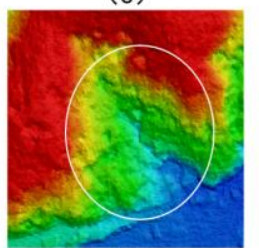

(h)

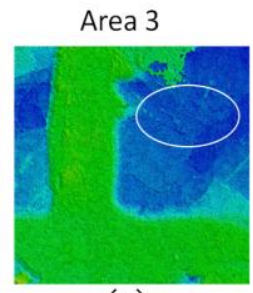

(c)

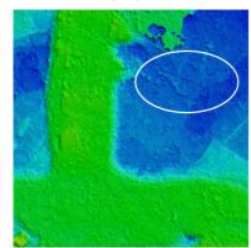

(f)

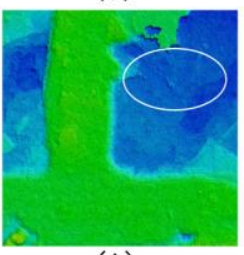

(i)
Figure 9. Comparison of median of grid cell and our method. (a), (b) and (c) are the raw data from dataset 1 and dataset 2; (d),

(e) and (f) are the results of median of grid cell and TIN algorithm; (g), (h) and (i) are the results of the proposed method.

\section{CONCLUSION}

In this paper, a novel semi-global merging of DSMs from multiple stereopairs is proposed to remove the inconsistency in the point cloud data. And experimental results show that DSM after merging is more accurate and reliable, and its data redundancy is effectively reduced. However, considering the lack of reference datasets, e.g., corresponding LiDAR data, more quantitative evaluation is still unavailable, which is also our further research.

\section{ACKNOWLEDGEMENTS}

This study was partially supported by Guangzhou city funding of science and technology (Project No. 201508020054), the funding of the workstation of Chinese Academicians by Guangdong province (Project No. 20130906) and China Postdoctoral Science Foundation funded project (Project No. 2016M602363). 


\section{REFERENCES}

Agrawal, M., Davis, L. S., 2001. A Probabilistic Framework for Surface Reconstruction from Multiple Images. In: IEEE Computer Society Conference on Computer Vision and Pattern Recognition, Kauai, HI, USA, Vol.2, pp. 470-476.

Amenta, N., Bern, M., 1999. Surface reconstruction by voronoi filtering. Discrete \& Computational Geometry, 22(4), pp.481504.

Axelsson, P., 2000. DEM generation from laser scanner data using adaptive TIN models. In: The International Archives of Photogrammetry and Remote Sensing, Amsterdam, Holland, Vol XXXIII, Part B4, pp. 110-117.

Fratarcangeli, F., Murchio, G., Rita, M. D., Nascetti, A. and Capaldo, P., 2016. Digital surface models from ZiYuan-3 triplet: performance evaluation and accuracy assessment. International Journal of Remote Sensing, (15), pp. 1-27.

Fuhrmann, S., Goesele, M., 2014. Floating scale surface reconstruction. $A C M, 33$ (4), pp.1-11.

Ghuffar, S., 2016. Satellite Stereo Based Digital Surface Model Generation Using Semi Global Matching in Object and Image Space. In: ISPRS Annals of the Photogrammetry, Remote Sensing and Spatial Information Sciences, Prague, Czech Republic, Vol. III-1, pp. 63-68.

Gong, K. and Fritsch, D., 2016. A Detailed Study about Digital Surface Model Generation Using High Resolution Satellite Stereo Imagery. In: ISPRS Annals of the Photogrammetry,
Remote Sensing and Spatial Information Sciences, Prague, Czech Republic, Vol. III-1, pp. 69-76.

Hirschmüller, H., 2005. Accurate and Efficient Stereo Processing by Semi-Global Matching and Mutual Information. Computer Vision and Pattern Recognition, 2, pp. 807-814.

Hirschmüller, H., 2008. Stereo Processing by Semiglobal Matching and Mutual Information. IEEE Transactions on Pattern Analysis and Machine Intelligence, 30(2), pp. 328-341.

Jaud, M., Passot, S., Bivic, R. L., Delacourt, C., Grandjean P. and Dantec, N. L., 2016. Assessing the Accuracy of High Resolution Digital Surface Models Computed by PhotoScan ${ }^{\circledR}$ and MicMac ${ }^{\circledR}$ in Sub-Optimal Survey Conditions. Remote Sensing, 8(6), pp. 465.

Kazhdan, M., Hoppe, H., 2013. Screened poisson surface reconstruction. ACM Transactions on Graphics, 32(3), pp.1-13.

Sadeq, H., Drummond, J. and Li, Z., 2016. Merging Digital Surface Models Implementing Bayesian Approaches. In: The International Archives of the Photogrammetry, Remote Sensing and Spatial Information Sciences, Prague, Czech Republic, Vol. XLI, Part B7, pp. 711-718.

Yastikli, N., Bayraktar, H. and Erisir, Z., 2014. Performance Validation of High Resolution Digital Surface Models Generated by Dense Image Matching with the Aerial Images. In: The International Archives of the Photogrammetry, Remote Sensing and Spatial Information Sciences, Denver, Colorado, USA, Vol. XL-1, pp. 429-433. 